\title{
$1 \quad$ A pipelining mechanism supporting previewing during visual exploration and reading
}

\author{
Ole Jensen $^{1 *}$, Yali Pan ${ }^{1}$, Steven Frisson ${ }^{1}$ and Lin Wang ${ }^{2,3}$
}

$3{ }^{1}$ Centre for Human Brain Health, School of Psychology, University of Birmingham, U.K.

$4{ }^{2}$ Department of Psychiatry and the Athinoula A. Martinos Center for Biomedical Imaging,

5 Massachusetts General Hospital, Harvard Medical School, Charlestown, Massachusetts

6 02129, US

$7 \quad{ }^{3}$ Department of Psychology, Tufts University, Medford, Massachusetts 02155, US

$8 \quad$ Corresponding author

\section{Abstract}

10 Humans have a remarkable ability to efficiently explore visual scenes and text by means of 11 eye-movements. Humans typically make eye-movements (saccades) every 250ms. Since the 12 saccadic motor planning and execution takes $100 \mathrm{~ms}$ this leaves only $\sim 150 \mathrm{~ms}$ to recognize the 13 fixated object (or word), while simultaneously previewing candidates for the next saccade 14 goal. We propose a pipelining mechanism that efficiently can coordinate visual exploration 15 and reading. The mechanism is timed by alpha oscillations that coordinate the saccades, 16 visual recognition and previewing in the cortical hierarchy. Consequently, the neuronal 17 mechanism supporting visual processing and saccades must be studied in unison to uncover 18 the brain mechanism supporting visual exploration and reading. 
21

22

24

\section{Highlights}

- Humans have a remarkable ability to efficiently acquire information from visual scenes and pages of text by means of saccadic exploration.

- Visual exploration is surprisingly efficient given the temporal and spatial constraints imposed by the visual system. As such, both information from current fixations as well as upcoming locations must be processed within a $150 \mathrm{~ms}$ time window.

- New data recording in humans and non-human primates points to a link between the timing of saccades and alpha oscillations.

- We present a framework in which visual exploration and reading are supported by similar neuronal mechanisms.

- We propose a novel mechanism in which visual exploration and reading is supported by a pipelining mechanism organized by alpha oscillations.

- According to the pipelining mechanism, fixated and previewed objects/words are represented at different phases of an alpha cycle. 
Our understanding of the visual system presents an intriguing conundrum: How do we manage to efficiently explore visual scenes and text by eye-movements given the relative slow and spatially limited processing capabilities of the human visual system? We saccade every $250-300 \mathrm{~ms}$ when reading and visually exploring natural scenes. Given that it takes about $100 \mathrm{~ms}$ to initiate and execute a saccadic motor program, there is only $150-200 \mathrm{~ms}$ available to process the fixated object or word while in parallel planning the next saccade. Importantly, since saccades typically land on informative objects or words [1,2] (Figure 1), a parafoveal previewing process is required when exploring and deciding on the next saccade goal. Thus, ultra-fast neuronal computation is essential for supporting saccadic exploration; however, we still need to uncover how the visual system can achieve this remarkable computational feat. The fast computation must rely on a highly tuned machinery in which eye-movements are coordinated with visual input [3]. As such, even small maladaptations can exacerbate problems in visual perception and possibly result in reading disorders.

The temporal constraints during visual exploration and reading

As mentioned above, the identification of current objects (pre-targets) as well as the saccade decision on future saccade goals (targets) must be made within $150 \mathrm{~ms}$ after fixating on the current object. This is because saccades are initiated as often as every $250 \mathrm{~ms}$ and it takes about $100 \mathrm{~ms}$ to initiate and execute a saccadic program towards the target $[4,5]$. It takes about $60 \mathrm{~ms}$ for information to travel from the retina to the visual cortex leaving about $90 \mathrm{~ms}$ for neocortical processing of the fixated object (Figure 2A-B). What is the evidence that visual objects can be identified within $150 \mathrm{~ms}$ ? Multivariate approaches applied to MEG data allow for identifying the neuronal fingerprint associated with representation of semantic features [6]. It was found that naturalness and animacy can be decoded from multivariate brain activity at respectively $122 \mathrm{~ms}$ and $157 \mathrm{~ms}$ after stimulus onset [6]. This timing is consistent with intracranial recordings in monkeys in which object category was decoded within $125 \mathrm{~ms}$ in the inferior temporal cortex [7]. Relating visual input to existing memory representations engaged the medial temporal lobe (i.e. parahippocampal areas, entorhinal cortex and hippocampus) at 150 - $200 \mathrm{~ms}$ after stimulus onset (for a review see [8]). In humans, event-related potentials increase in response to objects embedded in inconsistent compared to consistent scenes at about $300-400 \mathrm{~ms}$ (an N400 type response) [9-11]. However, this ERP response is so late that it cannot reflect early recognition. In short, electrophysiological studies suggest that it is possible to identify visual objects at the 
semantic level ("meaning") within $150 \mathrm{~ms}$. However, within the same $150 \mathrm{~ms}$ time-window future saccade goals must be explored and selected. Given that several processes must be completed within this short time-window, this poses a serious computational challenge to the visual system (Figure 2B). Parafoveal previewing prior to the saccade can serve to reduce recognition of the fixated object to about $110 \mathrm{~ms}[4,5,12]$. This then leaves $\sim 40 \mathrm{~ms}$ more for previewing the upcoming saccade goal (Figure 2C); i.e. it buys time to alter the saccade plan if, for instance, the saccade goal is deemed uninformative. We suggest that the acceleration of visual processing by previewing might be essential for efficient visual exploration. The temporal constraints during reading are equally tight. After the retinal input has arrived in occipital cortex at $60 \mathrm{~ms}$, the visual word form area (VWFA) is engaged for orthographic processing at about $90 \mathrm{~ms}$ [13] (Figure 2D-E). Later follows lexical access and semantic recognition supported by different parts of the left temporal cortex [13]. Eye-tracking research has demonstrated that fixation times are longer for low-compared to high-frequency words $[12,14]$ and that the word-frequency effect is present a least within $150 \mathrm{~ms}$ as revealed by survival analysis $[15,16]$. This implies that lexical identification completes before the motor program of the next saccade is initiated [4]; i.e. within $150 \mathrm{~ms}$ (Figure 2E). Electrophysiological findings quantifying ERPs and ERFs also support the notion that lexical identification happens within $150 \mathrm{~ms}$ [17-19]. As for natural vision, this presents an intriguing problem: if lexical access takes $150 \mathrm{~ms}$ and saccade programming about $100 \mathrm{~ms}$, lexical retrieval of the fixated word as well as previewing the next word in the parafovea must be completed within $150 \mathrm{~ms}$ [4, 20] (Figure 2E). Previewing will serve to reduce the lexico-semantic recognition possibly to about $110 \mathrm{~ms}[4,5,12]$, which will leave time to preview the next saccade goal (Figure 2F). This allows just enough time to alter the saccade plan if the next word is deemed uninformative (e.g. the).

\section{The spatial constraints during visual exploration and reading}

The visual system also holds an interesting spatial conundrum: Visual acuity drops dramatically for parafoveal vision (2-5 degrees relative to the current fixation) while our eyes can still saccade to relevant (and not necessarily salient) parts of visual scenes $[1,2,21]$ (Figure 1). As for visual exploration of natural scenes, it is of great interest to consider the spatial perceptual span in relation to parafoveal visual acuity [22, 23]. Using gaze-contingent paradigms that occlude the peripheral view, it was demonstrated that the radius of the effective visual span is about 8 degrees [24]. Likewise, by applying an artificial scotoma that moves with the gaze, participants could still perform a visual search task when occluding up 
104 to 4.1 degrees of the field of view. Even peripheral gist information is extracted in this type of paradigm [25]. The relatively large visual span in combination with reduced acuity for parafoveal vision begs the question: in which detail do we process an object before we saccade to it? [26]. In the light of this question, it is debated whether objects are previewed at the semantic level [26, 27]. For instance, studies have shown that search times are faster for objects embedded in inconsistent visual scenes (e.g. a tube of toothpaste in the living room) as compared to consistent scenes (e.g. a tube of toothpaste in the bathroom) [28]; however, it is debated whether this reflects semantic previewing [29, 30]. A recent EEG study investigated fixation-related potentials (FRPs) in response to pre-target objects prior to

113 saccading to target objects that were embedded in either consistent or inconsistent scenes.

114 Larger FRPs were found for objects embedded in inconsistent as compared to consistent 115 scenes [31]. Specifically a larger negative potential at $300 \mathrm{~ms}$ (akin to the N400 type ERP 116 effect) was observed in response to fixations at the pre-target object when the target was inconsistent with the scene. This finding provides support for semantic previewing.

119 As for reading, the spatial perceptual span in relation to parafoveal visual acuity has been 120 investigated using gaze-contingent paradigms in which performance is measured when the 121 text is occluded to the left and/or right of the gaze. This results in the conclusion that the 122 visual span extends 14-15 letter spaces (2-3 words) to the right of fixation and 3-4 letters to 123 the left [32]. Interestingly, this effect is reversed in readers of Hebrew who read from right to 124 left [33]. Therefore, the spatial perceptual span in reading is also not constrained by the reduction in visual acuity of extra-foveal vision. However, it has been shown that occluding the word just to the right of fixation, reduces reading-speed by $25-40 \mathrm{~ms}$ per word [12]. Interestingly, making the fixated word disappear after $60 \mathrm{~ms}$ hardly impacts reading, but making the parafoveal word disappear after $60 \mathrm{~ms}$, increases reading times substantially [34]. As such there is strong evidence that fluent reading relies on previewing.

130 Again, in which detail is upcoming text previewed in the parafovea? There is strong evidence 131 for previewing at the sub-lexical level (e.g. orthographic and phonological levels).

132 Experiments using the gaze-contingent boundary paradigm demonstrated that fixation times 133 on a word were reduced after it was primed in the parafovea by an orthographically similar 134 letter string (e.g sorp priming song) compared to an unrelated condition (door replaced by 135 song) (e.g [35-37]). A similar effect was found with respect to phonological previewing using 136 homophones (e.g., beach priming beech) [38, 39]. Previewing at the phonological level is 
137 also supported by reading studies manipulating the spelling-sound regularity. It is well 138 established that fixation times are longer on words with irregular spelling-sound mappings.

139 However, this difference disappears when previewing is prevented in a gaze contingent 140 paradigm [40].

141 It remains debated if words are previewed at the lexical and semantic level. Previewing at the

142 lexical level has been investigated using sentences containing target words of low- or high-

143 lexical frequency. Several eye-tracking studies have found that pre-target fixation times are 144 not modulated by the lexical frequency of the target word, suggesting the absence of lexical 145 previewing [41]. We recently challenged this notion by combining eye-tracking with a rapid 146 frequency tagging paradigm. In this paradigm, we subliminally flickered the target words that 147 were of low- or high-frequency during natural reading. We found that when readers fixated 148 on the pre-target words, there was a stronger tagging response as measured by MEG when the 149 target words were of low- compared to high-frequency [42]. This finding provides support for 150 previewing at the lexical level. Another controversial topic is whether there is previewing at 151 the semantic level. This question was studied using boundary paradigms in which the target word is changed just as the participant saccades to it. When for instance the target word changed from tune to song, the fixation times on song were compared to when the target word changed from the unrelated word door to song. The fixation times on song did not differ between these two conditions when the sentences were presented in English, indicating

156 a lack of semantic preview benefits [43]. Surprisingly, studies using Chinese [44] and 157 German [45] have found evidence for semantic previewing, e.g. shorter fixation times on $d o g$ 158 when the previewed word was puppy compared to when it was desk (for the German study, this was possibly explained by the capitalization of nouns [46]). Another EEG study was

160 based on participants reading lists of nouns that were either semantically related or not. In 161 favour of no semantic previewing [47], the fixation-related potentials for a given word did 162 not depend on whether the preceding word was semantically related to it or not. In sum, 163 while there is evidence for previewing at the sub-lexical level, there are mixed reports on 164 lexical and semantic previewing.

165 A mechanism supporting visual exploration and reading by pipelining coordinated by alpha oscillations

167 Having introduced the temporal and spatial constraints of the visual system, we here propose 168 a pipelining mechanism that can be used to guide efficient visual exploration and reading. 169 Before explaining the details of the mechanism, we will first review the temporal coding 
170 scheme observed in exploring animals that inspired the model. There is an intriguing link

171 between visual and spatial exploration. The goal of both behaviours is to process information

172 from the current location while deciding where to go next. Intracranial neuronal

173 investigations in behaving rats have provided important insights into the neurophysiological

174 mechanisms coordinating this process. Place cell recordings in the rat hippocampus have

175 demonstrated that neuronal theta oscillations $(6-12 \mathrm{~Hz})$ play an essential role for organizing

176 neuronal representations of space. The phenomenon of theta phase precession shows that a

177 given place cell fires late in the theta cycle as the rat enters a place field. As the rat advances,

178 the firing precesses to earlier theta phases. This finding is best explained by a mechanism in

179 which a sequence of spatial representations is 'read out' within a theta cycle [48, 49].

180 Neuronal representations early in the theta cycle code for current location whereas firing later

181 in the cycle codes for upcoming locations. This phase-coding scheme is consistent with a

182 pipelining mechanism in which different representations along the path are sequentially

183 processed at different theta phases. Could a related mechanism support visual exploration in which objects on a saccadic path are encoded as a sequence organized by oscillations?

186 In support of the above scheme, phase-coding with respect to neuronal oscillations has been

187 identified from intracranial recordings in humans performing visual and working memory 188 tasks. For instance, using human intracranial data it was demonstrated that individual 189 working memory representations are represented at different phases of an $8 \mathrm{~Hz}$ rhythm [50].

190 Another intracranial study found that different visual categories were reflected by a phase191 code [51]. Work based on intracranial recordings in non-human primates also reports phase coding in the visual system in various tasks [52-54]. Inspired by the phase-coding mechanism

193 we propose a framework (Box 1 and 2) where oscillations in the 8-13 Hz alpha band serve to 194 organize visual presentations in a phase-coded manner to support parafoveal previewing and 195 eventually guide the saccadic trajectory.

196 We hypothesize that natural visual exploration and reading relies on a process in which 197 several objects or words are processed simultaneously at different levels in the cortical 198 hierarchy. Consider Box 1A in which the viewer fixates on the woman. The visual input 199 propagates in the cortical hierarchy in which features of increasing complexity are combined 200 to eventually recognize the object woman in inferior temporal (IT) cortex. While the 201 participant fixates on the woman, the $d o g$ is previewed as a potential saccade goal. The 202 previewing creates a bottleneck problem in IT cortex when two objects (e.g. woman and $d o g$ ) 
are processed. We propose that the bottleneck-problem is solved by a pipelining mechanism in which several objects are processed simultaneously but at different levels in the cortical hierarchy (detailed in Box 1B). This scheme serves two different purposes: First, the preview of the $d o g$ will speed up the visual processing when this object eventually is fixated, thus reducing the recognition time. It is essential that the saccades are locked to the phase of the alpha oscillations in order for the processing to be coordinated. This scheme increases the efficacy of visual processing and it also buys some time allowing for the saccade plan to be revised in case the previewed object is deemed irrelevant.

211 We argue that a similar mechanism supports natural reading (see Box 2), with the exception

212 that the saccades typically are directed to the right. When the word jumped is fixated, this

213 allows for over to be previewed. To reduce a bottle-neck problem when processing the words,

214 they are organized in a temporal coding manner along the alpha cycle. Parafoveal previewing 215 results in the lexico-semantic identification reducing from about $150 \mathrm{~ms}$ to $110 \mathrm{~ms}$. This 216 provides extra time for evaluating the next saccade goal and potentially skip a less 217 informative word (e.g. the). Importantly, the saccades are locked to the phase of the alpha 218 oscillations in order to organize the timing of the processing and the visual input. In short, we 219 argue that efficient visual exploration and reading rely on parafoveal previewing, and the created bottleneck problem can be solved by a pipelining mechanism, suggesting that the processing of fixated and previewed objects is coordinated in time by alpha oscillations.

A computational mechanism organized as a pipeline requires an intricate temporal organization (Box 1 and 2). The transfer of representations between levels in the hierarchy as well as the sequential processing must be coordinated. In the example Box 1, some of the visual features of the boy will propagate to face-selective areas. Likewise, the face-selective area will process the boy slightly earlier than the woman. Based on recent findings, we propose that the oscillatory coupling serves to coordinate the information transfer between regions $[55,56]$ as well as organizing the sequential processing in a phase-coded manner.

Which neuronal dynamics might support a pipelining mechanism coordinated by brain oscillations? Based on human and animal data, it has been proposed that theta and alpha oscillations are a consequence of pulses of inhibition [57-59]. At the peak of an inhibitory pulse, neurons are prevented from firing. As the inhibition ramps down over the cycle, the most excitable neurons will fire first, then the somewhat less excited neurons and so forth. As such, the pulses of inhibition implement a type of filter, ensuring that neuronal representations are activated sequentially according to excitability $[59,60]$. This mechanism 
can account for the theta phase precession in rats [57] and has been proposed to operate in the visual system [59]. In case of the visual system, fixated representations are more excited compared to parafoveal representations. This allows the foveal representation to overcome the inhibition earlier and thus activate earlier in the alpha cycle. The sorting of visual representations, according to excitability, is a crucial component in the proposed pipelining mechanism (Box 1 and 2). While we have put forward an example with 2 objects in each cycle, the scheme could be extended to 3-4 objects and to include more hierarchical levels.

243 Computational modelling has proposed mechanism for how phase-coded representations are exchanged between brain regions [55]. Indeed, intracranial recordings in non-human primates suggest that synchronization in the alpha band reflects forward communication in the extended visual system [61]. The phase of the alpha oscillations modulates the gamma band activity, which serves to segment the representations in the alpha cycle. This scheme allows for the exchange of phase-coded representations between brain regions [62]. We propose that alpha-band phase-synchronization in the cortical hierarchy supports the phase-coded pipelining scheme by coordinating the feedforward transfer of increasingly higher level representations.

Evidence linking alpha oscillations and saccades

253 As outlined in Box 1 and 2, we propose that alpha oscillations are involved in organizing a 254 pipelining mechanism supporting visual processing. While alpha oscillations for decades were thought to reflect idling or a state of rest [63], it is now evident that they are involved in numerous cognitive processes $[58,64,65]$. One key observation is that alpha oscillations are present during continuous visual presentation e.g. [66, 67]. More recently, both human and animal research has found an intriguing link between the phase of alpha oscillations and saccades. A study based on both MEG and intracranial human data showed that saccade onsets are locked to the phase of ongoing alpha oscillations when viewing natural images [68]. Importantly, the locking of saccades to alpha phase was stronger for pictures later remembered as compared to later forgotten. This suggests that visual information impact memory areas stronger when saccades are coordinated by the phase of alpha oscillations. This notion is further supported by a nonhuman primate study in which saccades produced a phase-reset of theta/alpha oscillations in the hippocampus; the magnitude of the phase-reset predicted memory encoding [69]. Using EEG, it was shown that saccadic reaction times relates to the phase of pre-saccadic alpha oscillations [70]. A recent study in non-human 
269 decoding of the focus of the attentional spotlight. Importantly, the spotlight explored the 270 visual space at a 7-12 Hz alpha rhythm ('attentional saccades') [71]. In another non-human 271 primate study, signals from the V4 receptive field of respectively current and future fixations 272 were coherent in the alpha band around the time of saccades [72]. Finally, saccadic 273 suppression is also related to alpha oscillations: recording sites in V4 associated with 274 peripheral vision increase in alpha power during saccades [73]. In short, strong evidence is 275 accumulating in support of an intimate connection between saccades and alpha oscillations.

276 These findings in humans and animals provide some support for the proposed mechanism for 277 visual exploration and reading (Box 1 and 2)

278 Previewing primes and speeds up visual recognition

279 Key to the mechanism that we propose is that previewing speeds up the identification of 280 objects in visual scenes as well as lexico-semantic retrieval during reading [42, 74, 75]. 281 Several models suggest that object and lexical recognition relies on attractor type networks 282 (e.g. [76-79]). The core idea is that incomplete representations can be reconstructed through 283 pattern completion in a network of neurons with recurrent connections. The time it takes for an attractor network to converge is dependent on the basin of attraction and the trajectory to be travelled. Priming can be thought of as guiding a neuronal representation closer to the attractor as studied in computational models [80]. We propose that parafoveal previewing serves to prime a given neuronal representation so that when the respective object or word is fixated, retrieval is facilitated, and identification is sped up. In short, attractor type networks can provide a physiologically compatible account for how parafoveal previewing can prime object identification and lexico-semantic retrieval.

291 Concluding remarks

292 We argue that the visual system must operate in a highly efficient manner to support visual 293 exploration and reading. The core issue is that the fixated object or word must be processed 294 in the same time-interval as when the next saccade goal is planned. Given the bottleneck 295 problem in the visual hierarchy, we propose that this is achieved by a pipelining mechanism 296 in which current and upcoming spatial locations are processed - not simultaneously - but in 297 fast succession. Importantly, we propose that neuronal oscillations in the alpha band serve to 298 coordinate the pipelining mechanism that is implemented by a phase-coding scheme in which 299 different representations activate sequentially along the phase of the alpha oscillations. 300 Finally, to time the visual input with the neuronal processing, the mechanism also requires 301 that saccades are locked to the phase of the alpha oscillations. 
302 The framework results in a set of testable predictions. A core prediction is that alpha band 303 oscillations coordinate the neuronal processing associated with saccadic visual exploration.

304 As a result, representationally-specific representations for fixated and upcoming saccade 305 goals should be coupled to the phase of the alpha oscillations. This could be tested by MEG 306 or EEG recordings combined with eye-tracking studies in humans engaged in visual 307 exploration or reading tasks. The time-course of the representationally specific activation 308 could be identified by multivariate approaches and related to the phase of the ongoing alpha 309 oscillations [81]. As a complementary approach, rapid frequency tagging at different 310 frequencies (50 to $70 \mathrm{~Hz}$ ) could be used to track several objects and the respective neuronal 311 signals would then reflect the neuronal processing [42]. Specifically, we predict that the 312 fixated as well as the parafoveal object (or word) would be become active at different phases 313 of the alpha signal (see Box1C and Box2B). Importantly, we also predict that saccades would 314 be locked to alpha phase (as in [68]) and that this locking would be more pronounced with an 315 increase in task-demands. Finally, we predict that regions in the cortical hierarchy are phase316 locked in the alpha band. The phase-locking would allow for the phase-coded information to 317 be exchanged $[55,56]$; possibly there would be a systematic phase-shift of the alpha cycles 318 through the cortical hierarchy to guide the feed-forward flow of phase-coded information 319 [82].

320 In sum, we have here presented a novel and testable framework for the neuronal mechanisms 321 supporting visual exploration and reading in relation to saccades. Crucially, neuronal 322 oscillations are required for organizing the visual representation as well as the timing of 323 saccades. Since the proposed mechanism provides a unified account for visual exploration 324 and reading, it also opens the door for future investigations aimed at understanding the neuronal substrate associated with reading disorders. 


\section{Outstanding Questions}

328 In which detail are upcoming objects previewed before saccades are made to them? Are they previewed at the semantic level or maybe just in terms of features?

- In which detail are upcoming words previewed during reading before saccades are made to them? Are they primarily previewed at the sub-lexical (e.g. orthographical, phonological, orthographic) or also at the lexical and semantic level?

- What is the role of brain oscillations in visual exploration and reading? Recent studies have found cases where saccades are locked to the phase of alpha oscillations [68], but how general is this phenomenon?

- Are different objects and words represented along the phase of oscillations in the alpha cycle during visual exploration and reading (akin to the coding scheme of place representation organized by theta oscillations observed in exploration rats)? This can be addressed using multi-variate methods applied to MEG and EEG data in order to relate representational specific information to the phase of oscillations in the alpha band [81]. Likewise, rapid frequency tagging can be used to investigate the allocation of visual resources already prior to a saccade in relation to brain oscillations [42].

- Is there a link between previewing abilities and reading disorders? For instance, impaired previewing during reading might account for some of the reading deficits observed in some types of developmental and acquired dyslexia. Can our proposed pipelining mechanism account for the impaired previewing?

- Recent evidence suggests that prediction plays an important role in language comprehension [83-85]. How do top-down predictions impact bottom-up parafoveal previewing during reading? Could it be that both pre-activated representations and the representations of the previewed objects are encoded at the same phase of the alpha cycle? 
A)

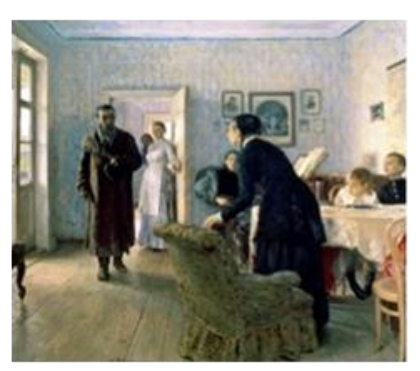

B)

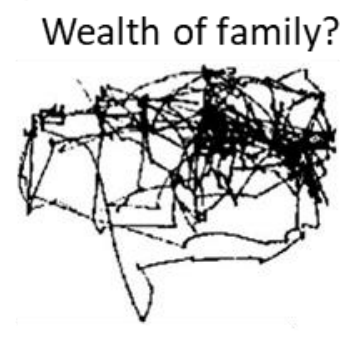

C)

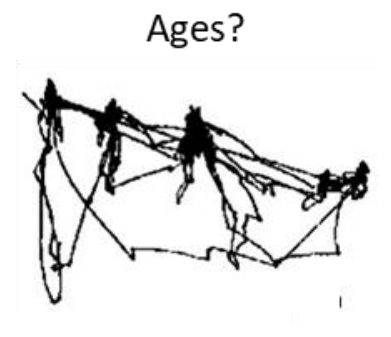

356 Figure 1. A) When exploring a picture we typically saccade 3-4 times per second. The classic

357 work by Yarbus (1967) demonstrates that saccades land on informative parts of the picture.

358 B) The saccadic path when the participant is asked to judge the wealth of the family. C) The

359 saccadic path when the participant is asked about the ages of the people in the picture. In

360 conclusion, objects explored and thus the saccadic path is depending on the goal of

361 exploration. These findings are consistent with the notion that objects in the scene are

362 previewed prior to saccading to them. Reproduced from Yarbus (1967) 
A)

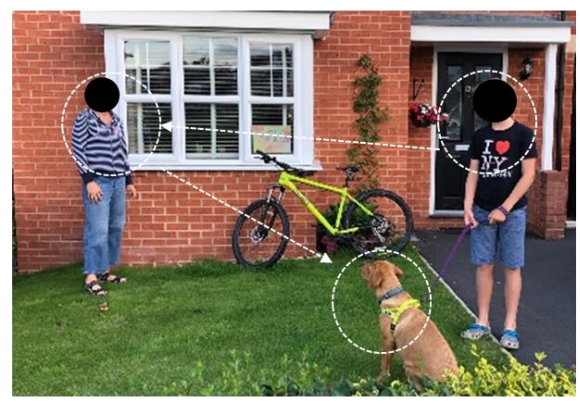

B) No preview benefit

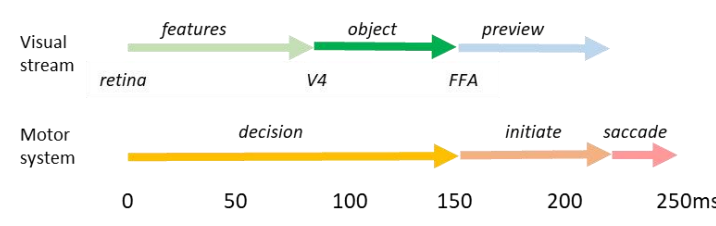

C) Preview benefit

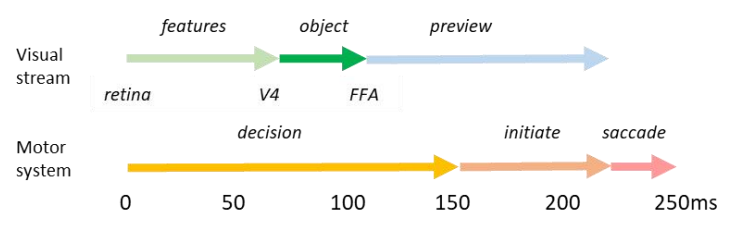

D)

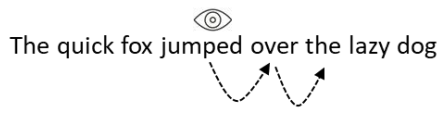

E) No preview benefit

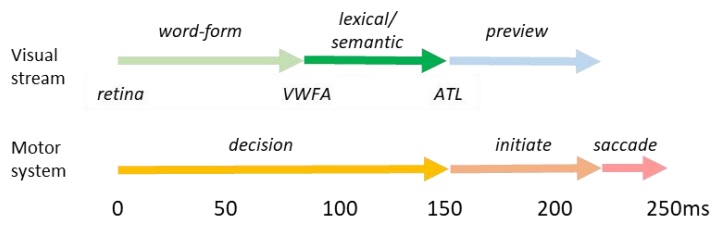

F) Preview benefit

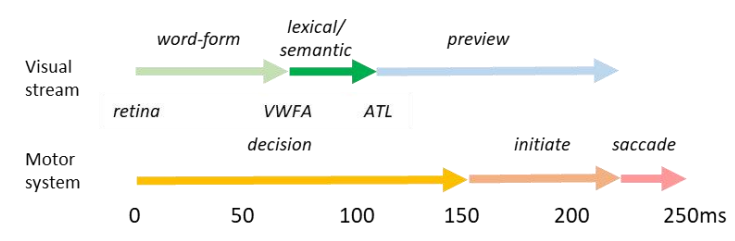

365 Figure 2. A) In this example the participant first fixates on the boy and then saccades to the 366 woman followed by the dog. B) The timing of the visual exploration process. The visual

367 object (the boy) arrives at $60 \mathrm{~ms}$ in V1 after which visual features are identified at about 85

$368 \mathrm{~ms}$. There is electrophysiological evidence suggesting that the object can be identified before

$369150 \mathrm{~ms}$ in object selective cortex. While this process is going on, the next saccadic decision

370 must be made (woman or dog?) such that the motor program can commence. It takes about

$371100 \mathrm{~ms}$ to initiate and execute the saccadic motor command. Typically, a saccade is executed

372 about $250 \mathrm{~ms}$ after the fixation onset. As both the object identification as well as the saccade

373 decision must be performed with $150 \mathrm{~ms}$, this places serious computational demands on the

374 visual system. C) Previewing by parafoveal processing allows for speeding up visual

375 processing. For instance, when fixating on the boy the woman can be previewed. When the

376 woman then is fixated the recognition can be reduced to about $110 \mathrm{~ms}$. This has two

377 important advantages: 1) it leaves about $40 \mathrm{~ms}$ for previewing the next saccade goal (the $\operatorname{dog}$ )

378 and 2) the preview occurs sufficiently early to impact the next saccade goal (e.g. to skip). D)

379 A sentence is read by fixating on the words sequentially. When fixating the word jumped it

380 must simultaneously be decided on whether to saccade to over. E) The timing of the visual

381 reading process. For instance, visual features of the word over arrive at $60 \mathrm{~ms}$ in V1 after

382 which the word-form is identified in the visual word-form area (VWFA) at $~ 90$ ms. There is

383 electrophysiological evidence suggesting that lexical recognition of the word is done within 
$384150 \mathrm{~ms}$ by a network including the anterior temporal lobe (ATL). While this process is going

385 on, the next saccade decision (over) must be made such that the saccadic motor program can

386 be initiated. Both the lexical identification as well as the saccade decision must be performed

387 within $150 \mathrm{~ms}$. F) Parafoveal previewing of a word (e.g. over) allows for reducing the lexico-

388 semantic identification upon fixation. As such a previewed fixated word could be recognized

389 at $110 \mathrm{~ms}$. This has an important advantage: it leaves more time for previewing the next word

390 and decide the next saccade goal before executing the saccade plan. For instance, a decision

391 might be made to skip highly common but uninformative words. 
A)
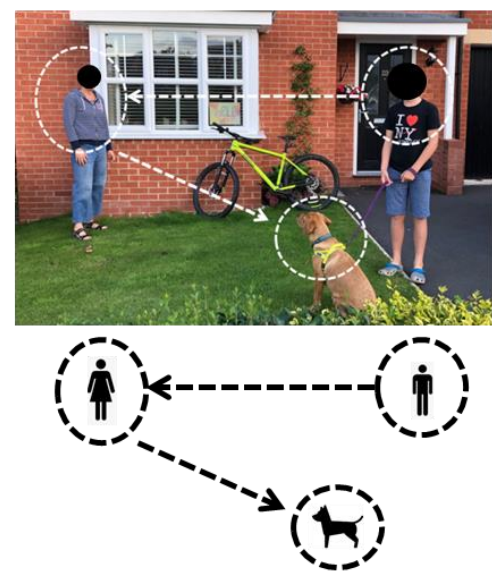

B)

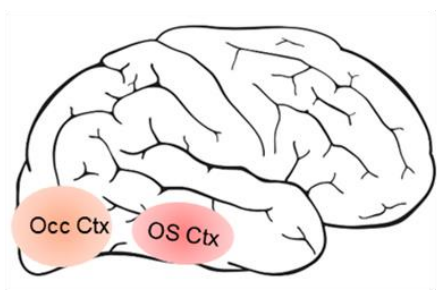

C)

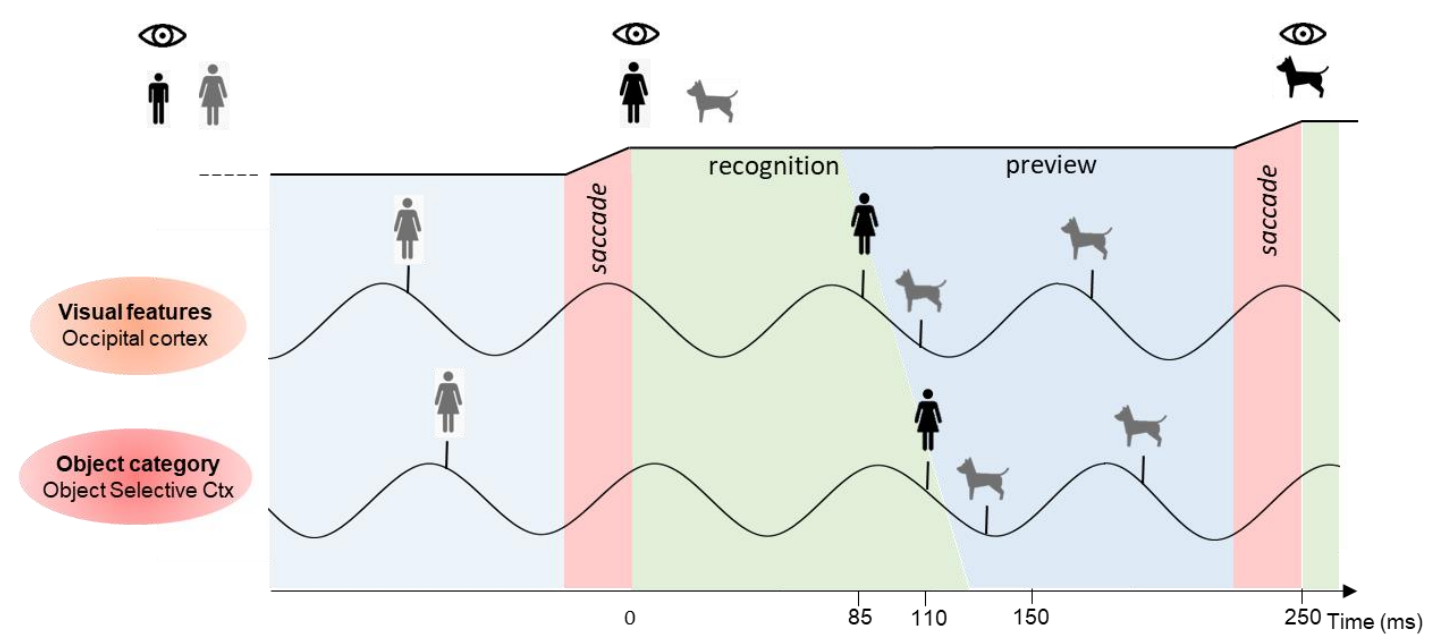

Box 1: A model for pipelining during visual exploration. A) The participant fixates on the

boy and then saccades to the woman and finally to the $d o g$. B) For simplicity, we assume two stages in which simple features (e.g. color) are identified first, followed by object category recognition in respectively V4 in occipital cortex and objects selective cortex in the inferior temporal lobe. C) We hypothesize that the temporal organization supporting the pipelining mechanism is coordinated by oscillations in the alpha band. In this example, $12 \mathrm{~Hz}$ alpha oscillations can be considered pulses of inhibition repeated every $83 \mathrm{~ms}$. At the peak of the alpha cycle, neuronal firing is inhibited. As the inhibition ramps down the most excitable representation will activate and so forth. Consider time point $\mathrm{t}=0 \mathrm{~ms}$ in which the participant moves the eyes from the boy and fixates on the woman. We assume that saccades are locked to the phase of the alpha oscillations such that the visual input of the woman arrives at the early down-going inhibitory slope of the alpha cycle at about $\mathrm{t}=85 \mathrm{~ms}$ where simple visual features of the woman engage visual occipital cortex (e.g. colors in V4). These feature representations are projected to object selective cortex for category identification by $110 \mathrm{~ms}$.

408 This fast category identification is made possible by the preview of the woman prior to the 409 saccade which has primed the 'semantic' access. Importantly, the pipelining scheme allows 
410 the woman and the $d o g$ to be processed in the same cycle in a multiplex manner thus avoiding

411 bottleneck problems. This scheme allows for a fast decision to be made to either saccade to

412 the $d o g$ or hold the saccade and preview another object as a potential target.

413

414 
A)

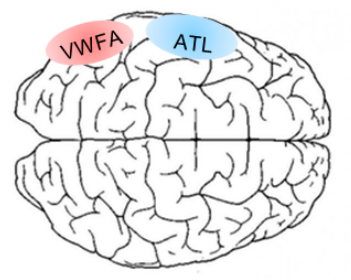

B)

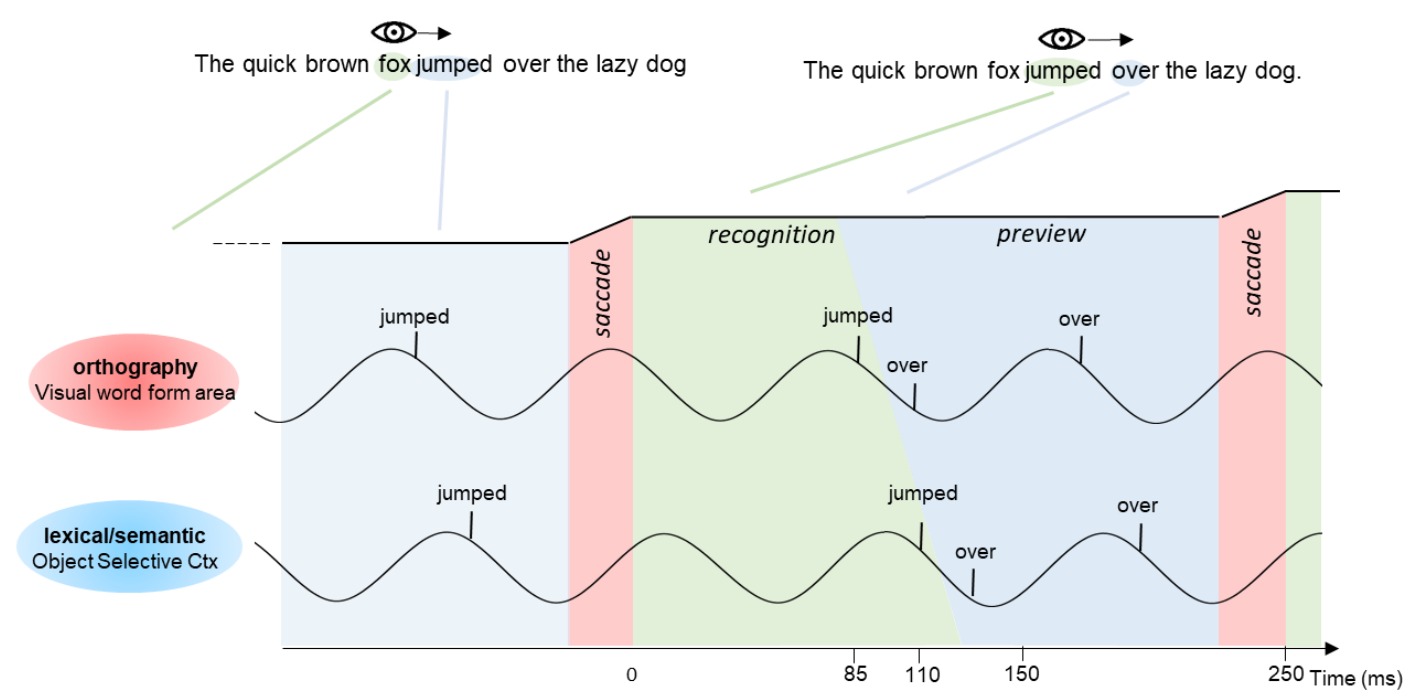

Box 2: A model for pipelining during natural reading. A) For the sake of simplicity, two

417 stages of word recognition are assumed, namely orthographic identification in visual word

418 from area (VWFA) followed by lexico-semantic access in an extended network including the

419 anterior temporal lobe. B) We hypothesize that the temporal organization supporting the

420 pipelining mechanism is coordinated by oscillations in the alpha band. The $12 \mathrm{~Hz}$ alpha

421 oscillations can be considered pulses of inhibition repeated every $83 \mathrm{~ms}$ [58]. Consider time

422 point $\mathrm{t}=0 \mathrm{~ms}$ in which the subject saccades and fixates on jumped. We assume that saccades

423 are locked to the phase of the alpha oscillations such that the visual input of jumped arrives at

424 the early down-going inhibitory slope of the alpha cycle at about $85 \mathrm{~ms}$ for orthographic

425 feature identification in the VWFA. The orthographic representations propagate to the ATL

426 for lexico-semantic identification by $110 \mathrm{~ms}$. This fast lexico-semantic process is made

427 possible by the preview of jumped prior to the saccade which has primed the lexical access.

428 Importantly, the pipeline scheme allows both jumped and over to be lexico-semantically

429 processed in the same cycle but at slightly different points in time, thus avoiding a bottleneck

430 problems in the ATL. During the fixation of over the word the is previewed. Given that the

431 word the carries little information, a decision to skip can be made. However, this is only

432 possible if over has been previewed since this will speed up the processing of over leaving

433 more time to preview the. 


\section{References (100 max)}

1. Yarbus, A.L. (1967) Eye movements and vision, Plenum Press.

437 2. Torralba, A. et al. (2006) Contextual guidance of eye movements and attention in real-world scenes: the role of global features in object search. Psychol Rev 113 (4), 766-86.

440

3. Liversedge, S.P. and Findlay, J.M. (2000) Saccadic eye movements and cognition. Trends Cogn Sci 4 (1), 6-14.

441

442

4. Reichle, E.D. and Reingold, E.M. (2013) Neurophysiological constraints on the eye-mind link. Front Hum Neurosci 7, 361.

443

444

5. Becker, W. and Jurgens, R. (1979) An analysis of the saccadic system by means of double step stimuli. Vision Res 19 (9), 967-83.

445

6. Cichy, R.M. et al. (2014) Resolving human object recognition in space and time. Nat Neurosci 17 (3), 455-62.

447

448

7. Hung, C.P. et al. (2005) Fast readout of object identity from macaque inferior temporal cortex. Science 310 (5749), 863-6.

449 8. Mormann, F. et al. (2008) Latency and selectivity of single neurons indicate hierarchical processing in the human medial temporal lobe. J Neurosci 28 (36), 8865-72.

451 9. Ganis, G. and Kutas, M. (2003) An electrophysiological study of scene effects on object identification. Brain Res Cogn Brain Res 16 (2), 123-44.

453 10. Mudrik, L. et al. (2010) ERP evidence for context congruity effects during simultaneous object454 scene processing. Neuropsychologia 48 (2), 507-17.

455 11. Vo, M.L. and Wolfe, J.M. (2013) Differential electrophysiological signatures of semantic and 456 syntactic scene processing. Psychol Sci 24 (9), 1816-23.

457 12. Rayner, K. (1998) Eye movements in reading and information processing: 20 years of research. 458 Psychol Bull 124 (3), 372-422.

459 13. Carreiras, M. et al. (2014) The what, when, where, and how of visual word recognition. Trends 460 Cogn Sci 18 (2), 90-8.

461 14. Rayner, K. (2009) Eye Movements in Reading: Models and Data. J Eye Mov Res 2 (5), 1-10.

462 15. Reingold, E.M. et al. (2012) Direct lexical control of eye movements in reading: evidence from a survival analysis of fixation durations. Cogn Psychol 65 (2), 177-206.

464 16. Sheridan, H. and Reichle, E.D. (2016) An Analysis of the Time Course of Lexical Processing During Reading. Cogn Sci 40 (3), 522-53.

466

467

468

469

470 17. Sereno, S.C. et al. (2003) Context effects in word recognition: evidence for early interactive processing. Psychol Sci 14 (4), 328-33.

18. Assadollahi, R. and Pulvermuller, F. (2003) Early influences of word length and frequency: a group study using MEG. Neuroreport 14 (8), 1183-7.

19. Dambacher, M. et al. (2006) Frequency and predictability effects on event-related potentials during reading. Brain Res 1084 (1), 89-103.

20. Sereno, S.C. and Rayner, K. (2000) The when and where of reading in the brain. Brain and Cognition 42 (1), 78-81.

474 21. Liversedge, S.P. et al. (2013) The Oxford handbook of eye movements, 2013 edition edn., Oxford 475 University Press.

476 22. Nuthmann, A. and Henderson, J.M. (2010) Object-based attentional selection in scene viewing. J 477 Vis $10(8), 20$.

478 23. Pajak, M. and Nuthmann, A. (2013) Object-based saccadic selection during scene perception: 479 evidence from viewing position effects. J Vis 13 (5).

480 24. Nuthmann, A. (2013) On the visual span during object search in real-world scenes. Visual 481 Cognition $21(7), 803-837$.

482 25. Larson, A.M. and Loschky, L.C. (2009) The contributions of central versus peripheral vision to 483 scene gist recognition. J Vis 9 (10), 61 1-16. 
26. Wu, C.C. et al. (2014) Guidance of visual attention by semantic information in real-world scenes. Front Psychol 5, 54.

27. Treisman, A.M. and Gelade, G. (1980) Feature-Integration Theory of Attention. Cognitive 487 Psychology 12 (1), 97-136.

488 28. LaPointe, M.R.P. and Milliken, B. (2016) Semantically incongruent objects attract eye gaze when viewing scenes for change. Visual Cognition 24 (1), 63-77.

490 29. Vo, M.L.H. and Henderson, J.M. (2011) Object-scene inconsistencies do not capture gaze: evidence from the flash-preview moving-window paradigm. Attention Perception \& Psychophysics 73 (6), 1742-1753.

493 30. Underwood, G. (2009) Cognitive Processes in Eye Guidance: Algorithms for Attention in Image Processing. Cognitive Computation 1 (1), 64-76. 31. Coco, M.l. et al. (2020) Fixation-related Brain Potentials during Semantic Integration of ObjectScene Information. Journal of Cognitive Neuroscience 32 (4), 571-589.

32. Rayner, K. et al. (1980) Asymmetry of the effective visual field in reading. Percept Psychophys 27 (6), 537-44.

498

33. Pollatsek, A. et al. (1981) Asymmetries in the perceptual span for Israeli readers. Brain Lang 14 (1), 174-80.

34. Rayner, K. et al. (2006) Eye movements when reading disappearing text: the importance of the word to the right of fixation. Vision Res 46 (3), 310-23.

35. Rayner, K. et al. (1980) Integrating information across eye movements. Cogn Psychol 12 (2), 20626.

36. McConkie, G.W. and Zola, D. (1979) Is visual information integrated across successive fixations in reading? Percept Psychophys 25 (3), 221-4.

507 37. Rayner, K. et al. (1986) Against parafoveal semantic preprocessing during eye fixations in 508 reading. Can J Psychol 40 (4), 473-83.

509 38. Chace, K.H. et al. (2005) Eye movements and phonological parafoveal preview: effects of reading 510 skill. Can J Exp Psychol 59 (3), 209-17.

511 39. Pollatsek, A. et al. (1992) Phonological codes are used in integrating information across saccades in word identification and reading. J Exp Psychol Hum Percept Perform 18 (1), 148-62.

514 40. Sereno, S.C. and Rayner, K. (2000) Spelling-sound regularity effects on eye fixations in reading. Percept Psychophys 62 (2), 402-9. 41. Degno, F. et al. (2019) Parafoveal previews and lexical frequency in natural reading: Evidence from eye movements and fixation-related potentials. J Exp Psychol Gen 148 (3), 453-474. 42. Pan, Y.F., S.; Jensen, O. (2020) Lexical parafoveal processing in natural reading predicts reading speed. bioRxiv. 43. Rayner, K. et al. (2003) On the processing of meaning from parafoveal vision during eye fixations in reading. Mind's Eye: Cognitive and Applied Aspects of Eye Movement Research, 213-234.

44. Yan, M. et al. (2009) Readers of Chinese extract semantic information from parafoveal words. Psychon Bull Rev 16 (3), 561-6.

45. Hohenstein, S. et al. (2010) Semantic preview benefit in eye movements during reading: A parafoveal fast-priming study. J Exp Psychol Learn Mem Cogn 36 (5), 1150-70.

46. Rayner, K. and Schotter, E.R. (2014) Semantic preview benefit in reading English: The effect of initial letter capitalization. J Exp Psychol Hum Percept Perform 40 (4), 1617-28.

47. Dimigen, O. et al. (2012) Trans-saccadic parafoveal preview benefits in fluent reading: A study with fixation-related brain potentials. Neuroimage 62 (1), 381-393.

48. Skaggs, W.E. et al. (1996) Theta phase precession in hippocampal neuronal populations and the compression of temporal sequences. Hippocampus 6 (2), 149-72.

531 49. Jensen, O. and Lisman, J.E. (1996) Hippocampal CA3 region predicts memory sequences: 532 accounting for the phase precession of place cells. Learn Mem 3 (2-3), 279-87.

533 50. Bahramisharif, A. et al. (2018) Serial representation of items during working memory 534 maintenance at letter-selective cortical sites. PLoS Biol 16 (8), e2003805. 
51. Watrous, A.J. et al. (2015) Phase-amplitude coupling supports phase coding in human ECoG. Elife 4.

52. Kayser, C. et al. (2012) Analysis of slow (theta) oscillations as a potential temporal reference frame for information coding in sensory cortices. PLoS Comput Biol 8 (10), e1002717.

539 53. Montemurro, M.A. et al. (2008) Phase-of-firing coding of natural visual stimuli in primary visual 540 cortex. Curr Biol 18 (5), 375-80. 54. Turesson, H.K. et al. (2012) Category-selective phase coding in the superior temporal sulcus. Proc Natl Acad Sci U S A 109 (47), 19438-43. 55. Jensen, O. (2001) Information transfer between rhythmically coupled networks: reading the hippocampal phase code. Neural Comput 13 (12), 2743-61. 56. Bonnefond, M. et al. (2017) Communication between Brain Areas Based on Nested Oscillations. eNeuro 4 (2).

57. Mehta, M.R. et al. (2002) Role of experience and oscillations in transforming a rate code into a temporal code. Nature 417 (6890), 741-6.

549 58. Mazaheri, A. and Jensen, O. (2010) Rhythmic pulsing: linking ongoing brain activity with evoked responses. Front Hum Neurosci 4, 177.

59. Jensen, O. et al. (2014) Temporal coding organized by coupled alpha and gamma oscillations prioritize visual processing. Trends Neurosci 37 (7), 357-69.

60. Gips, B. et al. (2016) A biologically plausible mechanism for neuronal coding organized by the phase of alpha oscillations. Eur J Neurosci 44 (4), 2147-61.

61. Saalmann, Y.B. et al. (2012) The pulvinar regulates information transmission between cortical areas based on attention demands. Science 337 (6095), 753-6.

62. Quax, S. et al. (2017) Top-down control of cortical gamma-band communication via pulvinar induced phase shifts in the alpha rhythm. PLoS Comput Biol 13 (5), e1005519.

559 63. Pfurtscheller, G. et al. (1996) Event-related synchronization (ERS) in the alpha band--an electrophysiological correlate of cortical idling: a review. Int J Psychophysiol 24 (1-2), 39-46.

561 64. Van Diepen, R.M. et al. (2019) The functional role of alpha-band activity in attentional processing: the current zeitgeist and future outlook. Curr Opin Psychol 29, 229-238.

563

564 65. Palva, S. and Palva, J.M. (2007) New vistas for alpha-frequency band oscillations. Trends Neurosci 30 (4), 150-8.

565

566 66. Wang, L. et al. (2018) Language Prediction Is Reflected by Coupling between Frontal Gamma and Posterior Alpha Oscillations. J Cogn Neurosci 30 (3), 432-447. 67. VanRullen, R. and Macdonald, J.S. (2012) Perceptual echoes at $10 \mathrm{~Hz}$ in the human brain. Curr Biol 22 (11), 995-9. 68. Staudigl, T. et al. (2017) Saccades are phase-locked to alpha oscillations in the occipital and medial temporal lobe during successful memory encoding. PLoS Biol 15 (12), e2003404. 69. Jutras, M.J. et al. (2013) Oscillatory activity in the monkey hippocampus during visual exploration and memory formation. Proc Natl Acad Sci U S A 110 (32), 13144-9.

70. Drewes, J. and VanRullen, R. (2011) This is the rhythm of your eyes: the phase of ongoing electroencephalogram oscillations modulates saccadic reaction time. J Neurosci 31 (12), 4698-708. 71. Gaillard, C. et al. (2020) Prefrontal attentional saccades explore space rhythmically. Nat Commun $11(1), 925$.

72. Neupane, S. et al. (2017) Coherent alpha oscillations link current and future receptive fields during saccades. Proc Natl Acad Sci U S A 114 (29), E5979-E5985. 73. Zanos, T.P. et al. (2016) Mechanisms of Saccadic Suppression in Primate Cortical Area V4. J Neurosci 36 (35), 9227-39.

581 74. Payne, B.R. et al. (2019) Event-related brain potentials reveal how multiple aspects of semantic 582 processing unfold across parafoveal and foveal vision during sentence reading. Psychophysiology 56 583 (10), e13432.

584 75. Stites, M.C. et al. (2017) Getting ahead of yourself: Parafoveal word expectancy modulates the 585 N400 during sentence reading. Cogn Affect Behav Neurosci 17 (3), 475-490. 
586 76. Cree, G.S. et al. (1999) An attractor model of lexical conceptual processing: Simulating semantic 587 priming. Cognitive Science 23 (3), 371-414.

588 77. McLeod, P. et al. (2000) Attractor dynamics in word recognition: converging evidence from errors 589 by normal subjects, dyslexic patients and a connectionist model. Cognition 74 (1), 91-113.

590 78. Devereux, B.J. et al. (2018) Integrated deep visual and semantic attractor neural networks 591 predict fMRI pattern-information along the ventral object processing pathway. Sci Rep 8 (1), 10636.

592 79. Daelli, V. and Treves, A. (2010) Neural attractor dynamics in object recognition. Exp Brain Res $593203(2), 241-8$.

594 80. Brunel, N. and Lavigne, F. (2009) Semantic priming in a cortical network model. J Cogn Neurosci $59521(12), 2300-19$.

596 81. van Es, M.W.J. et al. (2020) Phasic modulation of visual representations during sustained 597 attention. Eur J Neurosci.

598 82. Bahramisharif, A. et al. (2013) Propagating neocortical gamma bursts are coordinated by 599 traveling alpha waves. J Neurosci 33 (48), 18849-54.

600 83. Wang, L. et al. (2018) Specific lexico-semantic predictions are associated with unique spatial and 601 temporal patterns of neural activity. Elife 7.

602 84. Wang, L. et al. (2020) Neural Evidence for the Prediction of Animacy Features during Language 603 Comprehension: Evidence from MEG and EEG Representational Similarity Analysis. J Neurosci 40 604 (16), 3278-3291.

605 85. Kuperberg, G.R. and Jaeger, T.F. (2016) What do we mean by prediction in language 606 comprehension? Lang Cogn Neurosci 31 (1), 32-59. 